\title{
Triglyceride glucose-body mass index in identifying high-risk groups of pre-diabetes
}

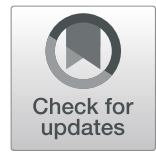

\author{
Chunyuan Jiang ${ }^{1}$, Ruijuan Yang ${ }^{2}$, Maobin Kuang ${ }^{3}$, Meng $\mathrm{Yu}^{1}$, Mingchun Zhong ${ }^{1}$ and Yang Zou $^{3^{*}}$ (D)
}

\begin{abstract}
Background: Triglyceride glucose-body mass index (TyG-BMI) has been recommended as an alternative indicator of insulin resistance. However, the association between TyG-BMI and pre-diabetes remains to be elucidated.

Methods: More than 100,000 subjects with normal glucose at baseline received follow-up. The main outcome event of concern was pre-diabetes defined according to the diagnostic criteria recommended by the American Diabetes Association (ADA) in 2018 and the World Health Organization (WHO) in 1999. A Cox proportional hazard regression model was used to evaluate the role of TyG-BMI in identifying people at high risk of pre-diabetes.
\end{abstract}

Results: At a mean observation period of 3.1 years, the incidence of pre-diabetes in the cohort was 3.70 and $12.31 \%$ according to the $\mathrm{WHO}$ and ADA diagnostic criteria for pre-diabetes, respectively. The multivariate Cox regression analysis demonstrated that TyG-BMI was independently positively correlated with pre-diabetes, and there was a special population dependence phenomenon. Among them, non-obese people, women and people under 50 years old had a significantly higher risk of TyG-BMI-related pre-diabetes ( $P$-interaction< 0.05$)$.

Conclusions: These findings suggest that a higher TyG-BMl significantly increases an individual's risk of pre-diabetes, and this risk is significantly higher in women, non-obese individuals, and individuals younger than 50 years of age.

Keywords: Pre-diabetes, TyG-BMI, World Health Organization, Triglyceride glucose-body mass index, American Diabetes Association

\section{Background}

Pre-diabetes is a subclinical high-risk state that progresses to diabetes and typical diabetes complications. It comprises two characteristics of impaired fasting glucose (IFG) and/or impaired glucose tolerance, and the glucose level is between normal and diabetic $[1,2]$. However, at present, there is no unified standard for an IFG-based diagnosis of pre-diabetes [1]. In the standards recommended by the World Health Organization (WHO), the definition of IFG uses fasting plasma glucose (FPG) in the range of $6.1-6.9 \mathrm{mmol} / \mathrm{L}$ [3], while the American Diabetes Association (ADA) uses a lower threshold for the definition of IFG (FPG: $5.6-6.9 \mathrm{mmol} / \mathrm{L}$ ) [4]. In the

\footnotetext{
* Correspondence: jxyxyzy@163.com

${ }^{3}$ From the Jiangxi Cardiovascular Research Institute, Jiangxi Provincial People's Hospital Affiliated to Nanchang University, Nanchang 330006, China Full list of author information is available at the end of the article
}

Chinese population, the prevalence of pre-diabetes diagnosed according to the WHO standard is $15.5 \%$, and that according to the ADA standard is $35.2 \%[5,6]$. In addition to the high prevalence of pre-diabetes, observational evidence shows that approximately $5-10 \%$ of patients with pre-diabetes develop diabetes each year $[1,2]$. On the other hand, pre-diabetes significantly increases the risk of macrovascular disease, retinopathy, autonomic neuropathy and kidney disease [7-10]. Therefore, early identification of modifiable risk factors is critical to reducing the long-term medical burden of pre-diabetes.

Previous studies have shown that insulin resistance (IR) is an important feature of the general population developing from normal glucose to pre-diabetes [11, 12], and the current gold standard for IR measurement is the hyperinsulinemic-euglycemic clamp (HEC) technique [13]. However, there are economic, time cost and ethical 
limitations in using HEC technology to evaluate IR during physical examinations in a large-scale population [14]. Therefore, as an alternative, some effective and inexpensive markers have been developed. Recently, a marker called the triglyceride glucose-body mass index (TyG-BMI) has received much attention. According to the description of Er et al., the ability of TyG-BMI to identify IR is better than that of other IR substitute markers [15]. In addition, some follow-up observational studies found that TyG-BMI has not only excellent discriminating ability in distinguishing IR but also good predictive performance in evaluating hypertension complicated with hyperuricemia, metabolic syndrome (MS), nonalcoholic fatty liver disease (NAFLD) and diabetes [16-19]. However, the longitudinal association between pre-diabetes and TyG-BMI remains to be studied. To address these issues, we retrospectively analysed the relationship between pre-diabetes and TyG-BMI in a large cohort in China according to the diagnostic criteria of pre-diabetes recommended by the WHO and ADA.

\section{Methods}

\section{Study design and subjects}

This is a post-hoc analysis of a large longitudinal cohort in China established by Rich Healthcare Group. The dataset of the analysis has been stored in the Dryad database by Chen et al. With reference to the terms of service of the database, researchers can reasonably apply data and indicate the source of data packets based on different research hypotheses [20]. In a previous study, Chen et al. analysed the association between age, body mass index (BMI) and diabetes [21], and the detailed study design was also described in a previous study [21]. In short, the original cohort of the study included all adults who underwent health examinations at Rich Healthcare Group in 11 major cities in China from 2010 to 2016. Subjects with at least two consecutive follow-up records were included. Subjects with incomplete measurements of height, weight and sex, unmeasured FPG, and extreme BMI values (subjects with BMI $>55 \mathrm{~kg} / \mathrm{m}^{2}$ or $<15 \mathrm{~kg} / \mathrm{m}^{2}$ were excluded, and the BMI range of 15$52.7 \mathrm{~kg} /{ }^{2}$ was allowed for inclusion in the study); diagnosed with diabetes at the baseline visit; followed up for less than 2 years and whose diabetes status could not be determined during follow-up were excluded. Finally, Chen et al. analysed the data of 211,833 people who underwent a physical examination. Our current study was a post-hoc analysis of the dataset shared by Chen et al., and we added the following exclusion criteria according to the diagnostic criteria of ADA and WHO for pre-diabetes: (1) subjects with incomplete lipid parameters; (2) subjects with $\mathrm{FPG} \geq 6.1 \mathrm{mmol} / \mathrm{L}$ (according to WHO diagnostic criteria) or $\geq 5.6 \mathrm{mmol} / \mathrm{L}$ (according to ADA diagnostic criteria) at the baseline visit; (3) subjects self-reporting diagnosed diabetes or FPG $>6.9$ during follow-up; and (4) subjects with missing FPG information during follow-up. Finally, 110,838 subjects could be used for analysis according to the diagnostic criteria of WHO for pre-diabetes and 100,309 subjects could be used for analysis according to the diagnostic criteria of ADA for pre-diabetes. Figure 1 shows a flow chart for study population inclusion. As the ethics committee of Rich Healthcare Group had authorized the previous study, the ethics committee of Jiangxi Provincial People's Hospital exempted the repeated application for ethical approval for this study (ethical review No. 2021-067).

\section{Data collection}

Trained health care workers recorded the baseline information of the subjects through standard questionnaires, including height, weight, age, sex, blood pressure, smoking and drinking history, family history and other biochemical indicators measured by physical examination. Height and weight measurements required subjects to take off their shoes and wear only lightweight clothes, with measurements accurate to $0.1 \mathrm{~kg}$ and $0.1 \mathrm{~cm}$. Analytical samples of FPG and other biochemical parameters were obtained at least $10 \mathrm{~h}$ after fasting and determined by a Beckman 5800 biochemical analyser. The same procedure was performed during the first visit and followup.

\section{TyG-BMI calculation}

TyG-BMI $=\mathrm{BMI} \times \mathrm{TyG}$ index, where $\mathrm{BMI}=$ weight $/$ height $^{2}$, and the TyG index $=\mathrm{Ln}[1 / 2 \mathrm{FPG}(\mathrm{mg} / \mathrm{dL}) \times \mathrm{TG}$ (mg/dL)] [15].

\section{Diagnosis of pre-diabetes}

In this study, we referred to the diagnostic criteria of IFG-based pre-diabetes recommended by the WHO in 1999 and ADA in 2018 [3, 4]; the WHO's IFG-based pre-diabetes diagnostic criteria were defined as FPG levels from 6.1 to $6.9 \mathrm{mmol} / \mathrm{L}$ [3], and the ADA's IFGbased pre-diabetes diagnostic criteria were defined as FPG levels from 5.6 to $6.9 \mathrm{mmol} / \mathrm{L}$ [4].

\section{Statistical analysis}

In this study, two sets of data were obtained after inclusion and exclusion according to the diagnostic criteria of pre-diabetes recommended by the WHO and ADA. The same steps were performed in both sets of data for all analyses. The statistical software for analysing the data were R language 3.4.3 and Empower (R) 2.20. The following outlines the specific statistical analysis in this study.

1. Description of baseline characteristics: First, the quartile of TyG-BMI was calculated by a quantile 


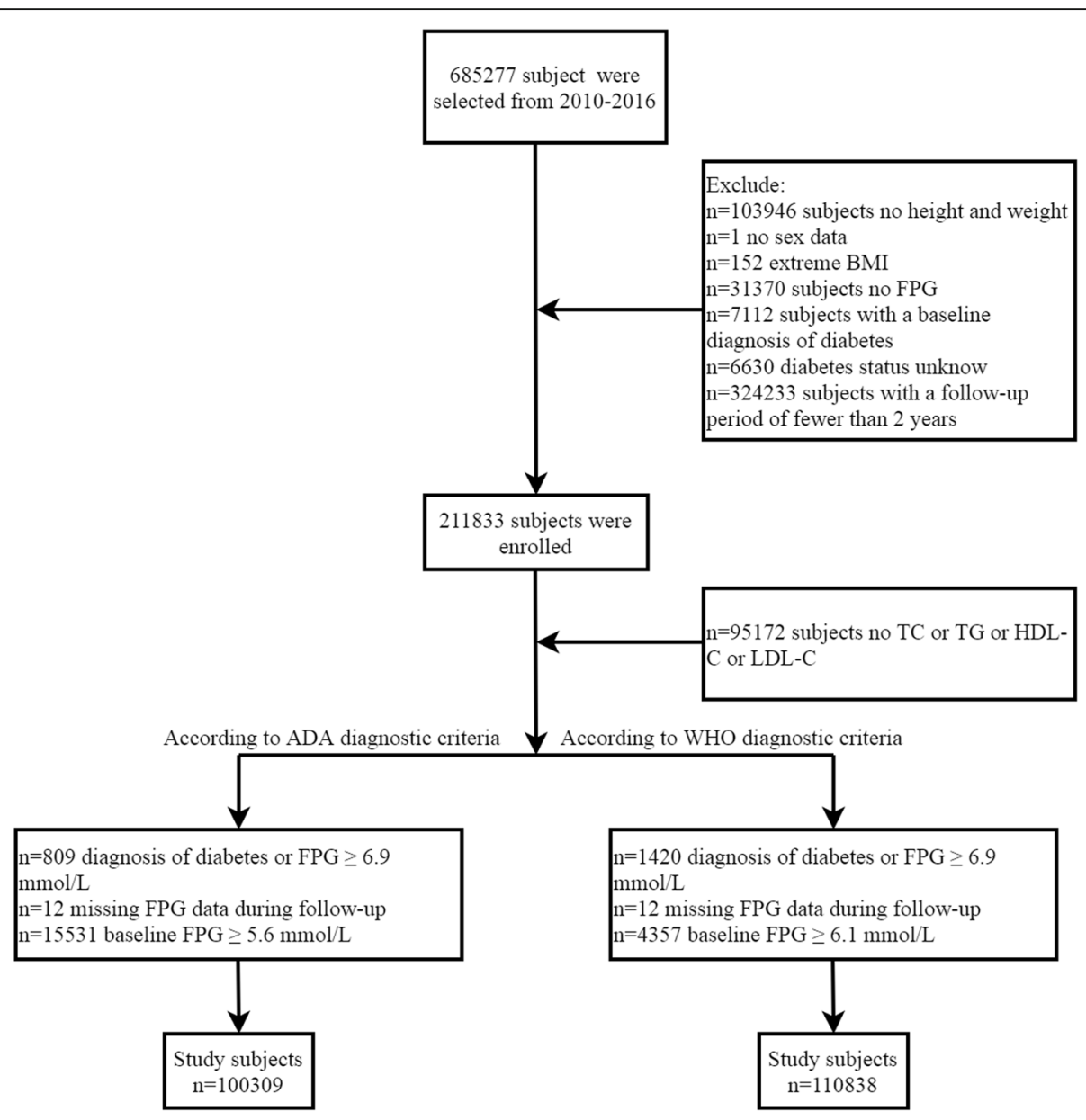

Fig. 1 Flow-chart of study selection process

function; then, the baseline data of subjects were stratified based on TyG-BMI quartiles and whether pre-diabetes was diagnosed; finally, the differences between groups were evaluated using a $t$ test, Kruskal-Wallis test, chi-squared test or one-way analysis of variance. Variables were described as percentages (\%), medians (quartile intervals) or means (standard deviations: SD).

2. Association analysis: A Cox proportional hazard regression model was used to evaluate the relationship between pre-diabetes and TyG-BMI, with hazard ratios (HR) and 95\% confidence intervals (CI) calculated. Before the model was established, the collinearity used by the variance inflation factor to evaluate covariates was calculated (Supplementary Table 1) [22]. Then, the cumulative incidence of pre-diabetic events with time was calculated by the Kaplan-Meier (KM) method, and whether it conformed to the proportional risk hypothesis of establishing the Cox model was determined by observing the KM curve corresponding to the TyG-BMI quartile. After following the above premise, we referred to the Strengthening the Reporting of Observational Studies in Epidemiology statement to implement the model adjustment strategy [23]. Model I adjusted for variables that had an impact of $>10 \%$ on the risk of pre-diabetes associated with TyGBMI [24]. Model II adjusted for covariates related to pre-diabetes + Model I. Model III adjusted for all non-collinear variables. The trend $P$ for TyG-BMI and pre-diabetes risk was examined in all three models by incorporating the TyG-BMI quartiles into the model as a continuous variable for analysis. Additionally, we also performed a receiver operating characteristic (ROC) curve analysis and calculated the best threshold and the area under the ROC curve (AUROC) for TyG-BMI for predicting prediabetes.

3. Subgroup analysis: This study conducted a stratified analysis based on the family history of diabetes, BMI, sex and age. Among them, the stratification of age refers to the research of Chen et al., and the classification was made according to decades [21]; 
the stratification of BMI refers to the standard recommended by the Chinese Obesity working Group, with $24 \mathrm{~kg} / \mathrm{m}^{2}$ as the cut-off point for overweight [25]. The stratified analysis was conducted through a Cox regression model, the differences between layers were compared by the likelihood ratio test, and the interaction was evaluated.

\section{Results}

\section{Clinical characteristics of subjects in the TyG-BMI quartile groups}

The dataset for inclusion and exclusion according to the WHO diagnostic criteria for pre-diabetes included 110,838 people (average age of 43.5 years old, $52.98 \%$ men, and $47.02 \%$ women). The dataset for inclusion and exclusion according to the ADA diagnostic criteria for pre-diabetes included 100,309 people (average age of 42.9 years, $51.97 \%$ men, and $48.03 \%$ women). In the two datasets included on the subjects according to ADA and WHO standards, we found significant differences in the clinical characteristics of the subjects among the quartiles of TyG-BMI (Table 1). Among them, the general measurement parameters, such as blood pressure, height, BMI and weight, in the group with high TyGBMI levels were higher than those in the group with low TyG-BMI levels. In addition, subjects with high levels of TyG-BMI generally had a higher proportion of men and older ages, with higher levels of FPG, creatinine $(\mathrm{Cr})$, total cholesterol (TC), aspartate aminotransferase (AST), low density lipoprotein cholesterol (LDL-C), blood urea nitrogen (BUN), triglyceride (TG) and alanine aminotransferase (ALT) and lower levels of high density lipoprotein cholesterol (HDL-C).

\section{Clinical characteristics of pre-diabetes}

At a mean observation period of 3.1 years, the incidence of pre-diabetes in the cohort was 3.70 and $12.31 \%$ according to $\mathrm{WHO}$ and ADA diagnostic criteria for pre-diabetes. The KM analysis showed a gradual increase in the cumulative incidence of prediabetes between the TyG-BMI quartile group (Fig. 2). Table 2 shows the clinical characteristics of individuals in the pre-diabetes group and the nonpre-diabetes group, and the results of the analysis of the two datasets were similar. Individuals with prediabetes were generally older; were more often male; had higher weight, BMI, SBP, and DBP; had higher levels of Cr, BUN, AST, ALT, LDL-C, TG, TC, and TyG-BMI; had lower levels of HDL-C; and were less likely to maintain smoking and drinking habits. These results suggest that patients with pre-diabetes appear to have a trend of metabolic disorders in an earlier period.

\section{Association of TyG-BMI with pre-diabetes}

The association of TyG-BMI with pre-diabetes was evaluated by a Cox regression model (Model I-III, Table 3). Regardless of the WHO or ADA diagnostic criteria for pre-diabetes, TyG-BMI was positively correlated with pre-diabetes in all three models $(P$-trend $<0.0001)$. Even after adjusting for all non-collinear variables, the regression coefficient of pre-diabetes risk corresponding to TyG-BMI was only slightly decreased (ADA: HR: 1.23, 95\% CI: 1.18-1.27; WHO: HR 1.40, 95\% CI: 1.32-1.47). Additionally, an ROC analysis was performed to evaluate the predictive value of TyG-BMI for pre-diabetes (Fig. 3). Table 4 shows the AUROC and best thresholds used by TyG-BMI, the TyG index and BMI to predict prediabetes. In the two datasets, the AUROC of TyG-BMI was the largest, showing moderate predictive performance for pre-diabetes.

\section{Subgroup analysis}

To explore whether there are differences in the risk for TyG-BMI-related pre-diabetes among different populations, a stratified analysis was also conducted. As shown in Table 5, higher levels of TyG-BMI among women, people under the age of 50, and non-obese people suggest a higher risk of developing pre-diabetes ( $P$-interaction $<0.05)$. In addition, although people with a family history of diabetes in the ADA group appeared to have a higher risk of developing pre-diabetes in the stratified analysis, further interaction tests suggested no significant difference $(P$-interaction $>0.05)$.

\section{Discussion}

This large retrospective cohort study demonstrated that over time higher TyG-BMI levels in healthy adults with normal glucose were positively correlated with newonset pre-diabetes. After further adjusting for age, sex, lifestyle factors, arterial blood pressure, and serological factors, the positive association between pre-diabetes and TyG-BMI remained stable. Additionally, the ROC analysis showed that TyG-BMI also had a moderate predictive value for pre-diabetes.

In recent years, with the advancement of urbanization, the nutrient intake ratio of residents has undergone a rapid change, accompanied by a significant decline in physical activity, and the glucose level of the global population is gradually rising [26]. From 1980 to 2008, the FPG of the whole population increased by approximately $0.1 \mathrm{mmol} / \mathrm{L}$, including $5.4 \mathrm{mmol} / \mathrm{L}$ in women and $5.5 \mathrm{mmol} / \mathrm{L}$ in men [27]. The incidence of pre-diabetes is increasing worldwide, and in China the prevalence of pre-diabetes according to the ADA diagnostic criteria has exceeded 35\% [1, 6].

At present, the diagnosis of pre-diabetes in China mostly refers to the standard recommended by ADA, 
Table 1 Baseline characteristics based on TyG-BMl grouping of subjects enrolled according to ADA and WHO diagnostic criteria for pre-diabetes

\section{TyG-BMI quartile}

According to WHO diagnostic criteria

\begin{tabular}{|c|c|c|c|c|c|}
\hline & Q1(96.68-168.15) & Q2(168.15-192.09) & Q3(192.09-218.70) & Q4(218.70-477.08) & $P$-value \\
\hline No. of subjects & 27,710 & 27,709 & 27,709 & 27,710 & \\
\hline Age, years & $35.00(31.00-43.00)$ & $40.00(33.00-51.00)$ & $44.00(35.00-55.00)$ & $45.00(36.00-56.00)$ & $<0.001$ \\
\hline Sex & & & & & $<0.001$ \\
\hline Men & 7394 (26.68\%) & $12,526(45.21 \%)$ & $17,684(63.82 \%)$ & $21,118(76.21 \%)$ & \\
\hline Women & 20,316 (73.32\%) & $15,183(54.79 \%)$ & 10,025 (36.18\%) & 6592 (23.79\%) & \\
\hline Height, cm & $164.16(7.50)$ & $165.43(8.30)$ & $167.12(8.48)$ & $168.41(8.31)$ & $<0.001$ \\
\hline Weight, kg & $52.76(6.02)$ & $60.48(6.93)$ & $67.47(7.70)$ & $77.44(10.06)$ & $<0.001$ \\
\hline $\mathrm{BMI}, \mathrm{kg} / \mathrm{m}^{2}$ & $19.54(1.37)$ & $22.04(1.19)$ & $24.10(1.30)$ & $27.24(2.32)$ & $<0.001$ \\
\hline $\mathrm{SBP}, \mathrm{mmHg}$ & 110.65 (13.57) & 116.14 (15.08) & $121.46(15.63)$ & $126.96(16.30)$ & $<0.001$ \\
\hline $\mathrm{DBP}, \mathrm{mmHg}$ & $69.22(9.19)$ & $72.06(9.83)$ & $75.64(10.35)$ & $79.59(11.06)$ & $<0.001$ \\
\hline $\mathrm{FPG}, \mathrm{mmol} / \mathrm{L}$ & $4.69(0.51)$ & $4.84(0.52)$ & $4.94(0.52)$ & $5.06(0.53)$ & $<0.001$ \\
\hline $\mathrm{TC}, \mathrm{mmol} / \mathrm{L}$ & $4.45(0.79)$ & $4.67(0.84)$ & $4.88(0.88)$ & $5.09(0.91)$ & $<0.001$ \\
\hline $\mathrm{TG}, \mathrm{mmol} / \mathrm{L}$ & $0.68(0.52-0.88)$ & $0.93(0.72-1.20)$ & $1.27(0.97-1.68)$ & $1.90(1.40-2.64)$ & $<0.001$ \\
\hline $\mathrm{HDL}-\mathrm{C}, \mathrm{mmol} / \mathrm{L}$ & $1.50(1.30-1.70)$ & $1.39(1.22-1.60)$ & $1.31(1.13-1.50)$ & $1.22(1.05-1.41)$ & $<0.001$ \\
\hline $\mathrm{LDL}-\mathrm{C}, \mathrm{mmol} / \mathrm{L}$ & $2.45(2.12-2.85)$ & $2.64(2.27-3.08)$ & $2.81(2.40-3.27)$ & $2.89(2.46-3.36)$ & $<0.001$ \\
\hline $\mathrm{ALT}, \mathrm{U} / \mathrm{L}$ & $13.00(10.30-17.00)$ & $15.90(12.00-22.00)$ & $20.00(15.00-28.00)$ & $27.50(19.10-40.40)$ & $<0.001$ \\
\hline AST, U/L & $20.00(17.00-23.00)$ & $21.00(18.00-25.00)$ & $22.50(19.00-27.00)$ & $25.00(21.00-30.80)$ & $<0.001$ \\
\hline BUN, mmol/L & $4.30(3.60-5.10)$ & $4.49(3.77-5.30)$ & $4.66(3.95-5.48)$ & $4.75(4.04-5.55)$ & $<0.001$ \\
\hline $\mathrm{Cr}$, umol/L & $60.70(53.60-71.50)$ & $66.40(56.40-79.20)$ & $73.00(61.00-83.40)$ & $76.20(65.90-85.80)$ & $<0.001$ \\
\hline TyG index & $7.84(0.38)$ & $8.19(0.39)$ & $8.52(0.42)$ & $8.97(0.50)$ & $<0.001$ \\
\hline Family history of diabetes & $571(2.06 \%)$ & $687(2.48 \%)$ & $583(2.10 \%)$ & $617(2.23 \%)$ & 0.004 \\
\hline Smoking status & & & & & $<0.001$ \\
\hline Non & $691(2.49 \%)$ & 1131 (4.08\%) & 1719 (6.20\%) & 2575 (9.29\%) & \\
\hline Past & $127(0.46 \%)$ & $234(0.84 \%)$ & $401(1.45 \%)$ & $474(1.71 \%)$ & \\
\hline Current & $6078(21.93 \%)$ & $5962(21.52 \%)$ & $5899(21.29 \%)$ & $5628(20.31 \%)$ & \\
\hline Not recorded & 20,814 (75.11\%) & $20,382(73.56 \%)$ & $19,690(71.06 \%)$ & 19,033 (68.69\%) & \\
\hline Drinking status & & & & & $<0.001$ \\
\hline Non & $63(0.23 \%)$ & $120(0.43 \%)$ & $225(0.81 \%)$ & $376(1.36 \%)$ & \\
\hline Past & $623(2.25 \%)$ & 1058 (3.82\%) & $1540(5.56 \%)$ & 1993 (7.19\%) & \\
\hline Current & $6210(22.41 \%)$ & $6149(22.19 \%)$ & $6254(22.57 \%)$ & $6308(22.76 \%)$ & \\
\hline Not recorded & $20,814(75.11 \%)$ & $20,382(73.56 \%)$ & $19,690(71.06 \%)$ & 19,033 (68.69\%) & \\
\hline \multicolumn{6}{|c|}{ According to ADA diagnostic criteria } \\
\hline & Q1(96.68-166.56) & Q2(166.56-189.88) & Q3(189.88-216.05) & Q4(216.06-477.08) & \\
\hline No. of subjects & 25,077 & 25,077 & 25,077 & 25,078 & \\
\hline Age, years & $35.00(31.00-42.00)$ & $39.00(33.00-49.00)$ & $43.00(35.00-54.00)$ & $44.00(36.00-55.00)$ & $<0.001$ \\
\hline Sex & & & & & $<0.001$ \\
\hline Men & $6500(25.92 \%)$ & $10,892(43.43 \%)$ & $15,657(62.44 \%)$ & $19,081(76.09 \%)$ & \\
\hline Women & $18,577(74.08 \%)$ & $14,185(56.57 \%)$ & $9420(37.56 \%)$ & $5997(23.91 \%)$ & \\
\hline Height, cm & $164.13(7.46)$ & $165.29(8.24)$ & $167.06(8.49)$ & $168.46(8.30)$ & $<0.001$ \\
\hline Weight, kg & $52.50(5.93)$ & $59.99(6.83)$ & $67.00(7.64)$ & $77.05(10.01)$ & $<0.001$ \\
\hline $\mathrm{BMI}, \mathrm{kg} / \mathrm{m}^{2}$ & $19.45(1.35)$ & $21.90(1.17)$ & $23.94(1.29)$ & $27.08(2.30)$ & $<0.001$ \\
\hline
\end{tabular}


Table 1 Baseline characteristics based on TyG-BMI grouping of subjects enrolled according to ADA and WHO diagnostic criteria for pre-diabetes (Continued)

\begin{tabular}{|c|c|c|c|c|c|}
\hline \multirow{2}{*}{\multicolumn{6}{|c|}{ According to WHO diagnostic criteria }} \\
\hline & & & & & \\
\hline & Q1(96.68-168.15) & Q2(168.15-192.09) & Q3(192.09-218.70) & Q4(218.70-477.08) & $P$-value \\
\hline $\mathrm{SBP}, \mathrm{mmHg}$ & $110.30(13.41)$ & $115.25(14.83)$ & $120.60(15.33)$ & $126.16(16.12)$ & $<0.001$ \\
\hline $\mathrm{DBP}, \mathrm{mmHg}$ & $69.05(9.15)$ & $71.61(9.76)$ & $75.19(10.22)$ & $79.16(11.06)$ & $<0.001$ \\
\hline $\mathrm{FPG}, \mathrm{mmol} / \mathrm{L}$ & $4.65(0.48)$ & $4.76(0.47)$ & $4.83(0.46)$ & $4.91(0.44)$ & $<0.001$ \\
\hline $\mathrm{TC}, \mathrm{mmol} / \mathrm{L}$ & $4.44(0.79)$ & $4.65(0.83)$ & $4.85(0.87)$ & $5.06(0.91)$ & $<0.001$ \\
\hline $\mathrm{TG}, \mathrm{mmol} / \mathrm{L}$ & $0.67(0.52-0.86)$ & $0.90(0.70-1.18)$ & $1.23(0.95-1.62)$ & $1.87(1.37-2.57)$ & $<0.001$ \\
\hline $\mathrm{HDL}-\mathrm{C}, \mathrm{mmol} / \mathrm{L}$ & $1.50(1.30-1.70)$ & $1.40(1.23-1.60)$ & $1.31(1.14-1.50)$ & $1.22(1.05-1.41)$ & $<0.001$ \\
\hline LDL-C, mmol/L & $2.44(2.11-2.84)$ & $2.63(2.26-3.05)$ & $2.79(2.37-3.24)$ & $2.87(2.45-3.34)$ & $<0.001$ \\
\hline$A L T, U / L$ & $13.00(10.30-17.00)$ & $15.40(11.90-21.30)$ & $19.60(14.50-27.80)$ & $27.00(19.00-40.00)$ & $<0.001$ \\
\hline AST, U/L & $20.00(17.00-23.00)$ & $21.00(18.00-24.60)$ & $22.00(19.00-26.60)$ & $24.90(20.90-30.20)$ & $<0.001$ \\
\hline $\mathrm{BUN}, \mathrm{mmol} / \mathrm{L}$ & $4.28(3.60-5.08)$ & $4.45(3.74-5.23)$ & $4.61(3.91-5.42)$ & $4.71(4.01-5.50)$ & $<0.001$ \\
\hline $\mathrm{Cr}$, ummol/L & $60.50(53.40-71.00)$ & 65.90 (56.00-78.80) & $72.70(60.80-83.00)$ & 76.20 (65.90-85.60) & $<0.001$ \\
\hline TyG index & $7.82(0.38)$ & $8.15(0.38)$ & $8.47(0.41)$ & $8.91(0.50)$ & $<0.001$ \\
\hline Family history of diabetes & $513(2.05 \%)$ & 607 (2.42\%) & $530(2.11 \%)$ & $558(2.23 \%)$ & 0.025 \\
\hline Smoking status & & & & & $<0.001$ \\
\hline Non & $614(2.45 \%)$ & 985 (3.93\%) & 1486 (5.93\%) & 2264 (9.03\%) & \\
\hline Past & $110(0.44 \%)$ & 198 (0.79\%) & 349 (1.39\%) & 433 (1.73\%) & \\
\hline Current & 5441 (21.70\%) & $5372(21.42 \%)$ & $5301(21.14 \%)$ & $5085(20.28 \%)$ & \\
\hline Not recorded & $18,912(75.42 \%)$ & $18,522(73.86 \%)$ & $17,941(71.54 \%)$ & 17,296 (68.97\%) & \\
\hline Drinking status & & & & & $<0.001$ \\
\hline Non & $56(0.22 \%)$ & $100(0.40 \%)$ & 177 (0.71\%) & 306 (1.22\%) & \\
\hline Past & $530(2.11 \%)$ & 928 (3.70\%) & 1323 (5.28\%) & 1767 (7.05\%) & \\
\hline Current & 5579 (22.25\%) & 5527 (22.04\%) & 5636 (22.47\%) & 5709 (22.76\%) & \\
\hline Not recorded & 18,912 (75.42\%) & 18,522 (73.86\%) & 17,941 (71.54\%) & 17,296 (68.97\%) & \\
\hline
\end{tabular}

Continuous variables were summarized as mean (SD) or medians (quartile interval), the differences among quartiles were evaluated by one-way analysis of variance and Tukey's HSD test or the Kruskal-Wallis test and Steel-Dwass test. After making a pairwise comparison between the quintiles, the results showed that there were significant differences among all groups $(P<0.05)$. Categorical variables were displayed as percentage $(\%)$

Abbreviations: ADA American Diabetes Association, WHO World Health Organization, BMI Body mass index, SBP systolic blood pressure, DBP diastolic blood pressure, FPG fasting plasma glucose, $T G$ triglyceride, $T y G$ the triglyceride-glucose index, $T y G-B M I$ triglyceride glucose-body mass index, $T C$ total cholesterol, $L D L-C$ low-density lipid cholesterol, ALT alanine aminotransferase, AST aspartate aminotransferase, BUN blood urea nitrogen, $C r$ creatinine

and some studies refer to the diagnostic standard of the WHO. The prevalence of pre-diabetes diagnosed according to the ADA criteria is also higher due to its lower diagnosis threshold for IFG-based pre-diabetes [7-10]. Considering that the definition of pre-diabetes is not uniform, a high threshold may lead to missed diagnosis in some high-risk individuals, while a lower threshold can easily lead to overdiagnosis. Therefore, in the same cohort, the diagnostic criteria of the WHO and ADA were referenced in this study to evaluate the association of pre-diabetes with TyG-BMI and to further verify the stability of the association between the two. At an average observation period of 3.1 years, there was a significant difference in the number of pre-diabetes diagnoses according to the two criteria, with an incidence of prediabetes diagnosed according to the WHO criteria of
$3.70 \%$ and an incidence of pre-diabetes diagnosed according to the ADA criteria of $12.31 \%$. The results of these differences were similar to those of previous surveys $[5,6]$.

\section{Comparisons with other studies and what does the current work add to the existing knowledge}

The triglyceride glucose (TyG) index is a combined marker containing TG and FPG, and it has been widely studied in the early period and is regarded as an alternative marker of IR $[28,29]$. In a recent study by Wen et al., the TyG index was found to also identify people at risk of pre-diabetes [30]. Their findings further suggest that IR is closely related to pre-diabetes. BMI is the simplest anthropometric indicator, and it is often used to assess obesity and the risk of metabolic diseases [31]. 

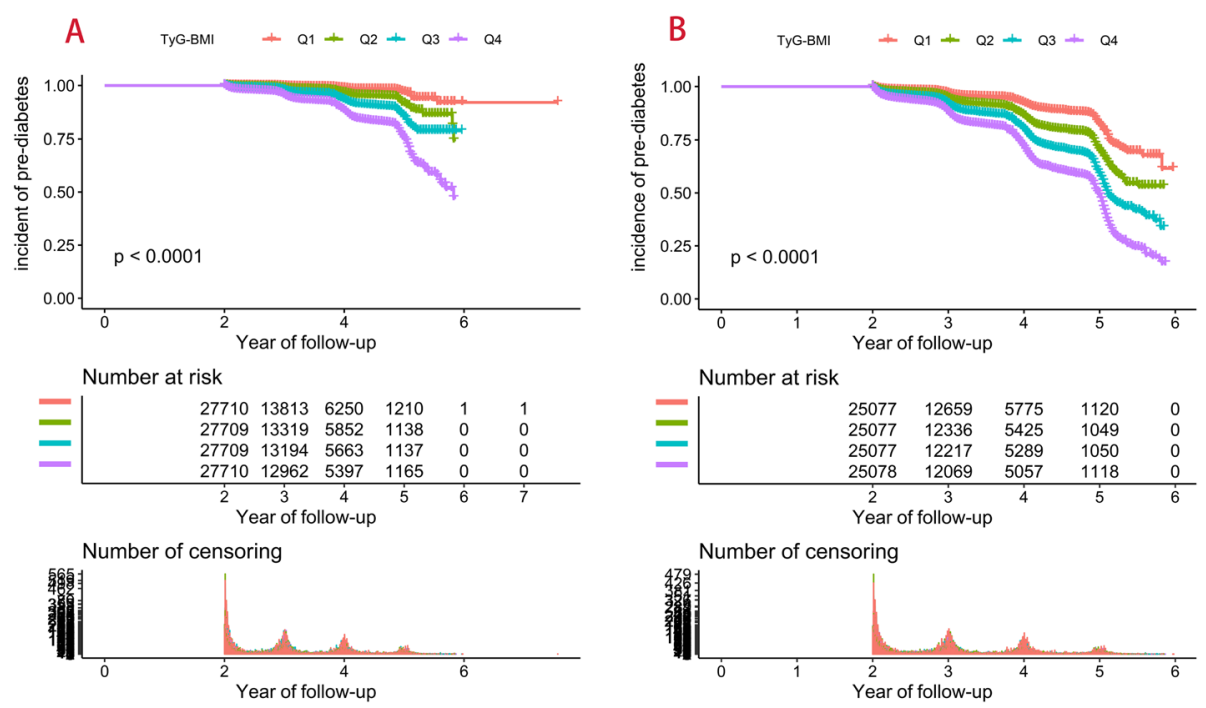

Fig. 2 Kaplan-Meier curve for pre-diabetes (A: according to WHO diagnostic criteria; B: according to ADA diagnostic criteria). TyG-BMI: triglyceride glucose-body mass index; WHO: World Health Organization; ADA: American Diabetes Association

TyG-BMI is a newly developed obesity-related parameter in recent years. It is the product of BMI and the TyG index. In the earliest related study of TyG-BMI, Er et al. found that TyG-BMI is a better predictor of IR than the TyG index, BMI, traditional lipids, lipid ratios, the visceral obesity index, the visceral adiposity index, lipid accumulation products, and adipose factors [15]. Recently, more evidence has shown that TyG-BMI is closely related to adverse metabolic characteristics, including abnormal serum uric acid, abnormal blood pressure, dyslipidemia and abnormal glucose [16-19]. In March 2021, Raimi et al. analysed the association of TyG-BMI with MS. They noted that the product of BMI and the TyG index improved the ability to identify and predict MS (AUROC $=0.838)$ [16]. In May, another study found that TyG-BMI also had a moderate ability to distinguish between hypertension and hyperuricemia [17]. Apart from that, several observational studies further found that TyG-BMI has the potential to predict the risk of NAFLD and diabetes, especially in young and middleaged people and non-obese people $[18,19]$. These TyGBMI-related results suggest that this parameter has the potential to be used as a marker of metabolic diseases. However, the current research on the association of prediabetes with TyG-BMI is limited, and there are only two related studies. Zheng et al. first found that TyGBMI can be used to assess the pre-diabetes risk of firstdegree relatives of diabetic patients [32], and a subsequent study in India found a similar association in the general population [33]. Although these two studies have revealed an association between TyG-BMI and prediabetes, there are still some limitations in their studies. (1) Follow-up data were not included in their study, so a longitudinal association could not be analysed. (2) Their study did not further analyse the differences in the relationship between the two groups in different populations. (3) Their sample size is relatively small (166 and 1544). (4) The diagnostic criteria for pre-diabetes were different in their study. To further explore the longitudinal association of pre-diabetes with TyG-BMI, this study included the sample data of more than 100,000 subjects and referred to the diagnostic criteria of the $\mathrm{ADA}$ and $\mathrm{WHO}$ at the same time. The results confirmed their conclusions and further found that the association between the two was significantly different in different populations. Additionally, we note that the risk factors for pre-diabetes, diabetes and cardiovascular disease in the Asian population are similar to those of other ethnic groups [34, 35]. These findings further imply that TyGBMI may be equally useful in cardiovascular disease risk assessment.

New evidence in this study suggests that in people with a family history of diabetes, the relationship between pre-diabetes and TyG-BMI based on ADA diagnostic criteria is significant. This result is consistent with the findings of Zheng et al. [32]; however, the results of further interactive tests in this study suggest that this finding is not significant ( $P$-interaction $>0.05)$. In addition to examining the correlation between prediabetes and TyG-BMI in people with a family history of diabetes, we also conducted an exploratory analysis in different age, sex, and BMI groups. Our evidence suggests that the TyG-BMI-related pre-diabetes risk was significantly higher in people younger than 50 years of age, women, and non-obese individuals. This finding is similar to the findings of subgroups of several recent 
Table 2 Baseline characteristics of the pre-diabetes and non-pre-diabetes groups

\begin{tabular}{|c|c|c|c|c|c|c|}
\hline & WHO & & $P$-value & ADA & & $P$-value \\
\hline & Non-Pre-diabetes & Pre-diabetes & & Non-Pre-diabetes & Pre-diabetes & \\
\hline No. of subjects & 106,735 & 4103 & & 87,957 & 12,352 & \\
\hline Age, years & $40.00(33.00-51.00)$ & $53.00(44.00-62.00)$ & $<0.001$ & $39.00(33.00-49.00)$ & $48.00(37.00-59.00)$ & $<0.001$ \\
\hline Sex & & & $<0.001$ & & & $<0.001$ \\
\hline Men & $56,105(52.56 \%)$ & 2617 (63.78\%) & & 44,395 (50.47\%) & 7735 (62.62\%) & \\
\hline Women & $50,630(47.44 \%)$ & 1486 (36.22\%) & & 43,562 (49.53\%) & 4617 (37.38\%) & \\
\hline Height, cm & $166.28(8.30)$ & $166.38(8.57)$ & 0.430 & $166.17(8.28)$ & $166.69(8.43)$ & $<0.001$ \\
\hline Weight, kg & $64.32(11.93)$ & $70.09(12.30)$ & $<0.001$ & $63.59(11.81)$ & $68.02(11.91)$ & $<0.001$ \\
\hline $\mathrm{BMI}, \mathrm{kg} / \mathrm{m}^{2}$ & $23.15(3.22)$ & $25.21(3.28)$ & $<0.001$ & $22.91(3.17)$ & $24.38(3.25)$ & $<0.001$ \\
\hline $\mathrm{SBP}, \mathrm{mmHg}$ & $118.38(16.11)$ & $129.91(18.46)$ & $<0.001$ & $117.08(15.61)$ & $125.17(17.52)$ & $<0.001$ \\
\hline $\mathrm{DBP}, \mathrm{mmHg}$ & $73.91(10.76)$ & $79.85(11.62)$ & $<0.001$ & $73.21(10.57)$ & $77.61(11.38)$ & $<0.001$ \\
\hline $\mathrm{FPG}, \mathrm{mmol} / \mathrm{L}$ & $4.86(0.53)$ & $5.38(0.48)$ & $<0.001$ & $4.75(0.47)$ & $5.03(0.40)$ & $<0.001$ \\
\hline $\mathrm{TC}, \mathrm{mmol} / \mathrm{L}$ & $4.76(0.89)$ & $5.05(0.93)$ & $<0.001$ & $4.72(0.88)$ & $4.92(0.90)$ & $<0.001$ \\
\hline $\mathrm{TG}, \mathrm{mmol} / \mathrm{L}$ & $1.08(0.74-1.60)$ & $1.50(1.00-2.20)$ & $<0.001$ & $1.02(0.72-1.52)$ & $1.30(0.90-1.94)$ & $<0.001$ \\
\hline $\mathrm{HDL}-\mathrm{C}, \mathrm{mmol} / \mathrm{L}$ & $1.35(1.17-1.57)$ & $1.32(1.12-1.52)$ & $<0.001$ & $1.36(1.17-1.58)$ & $1.32(1.14-1.53)$ & $<0.001$ \\
\hline LDL-C, mmol/L & $2.68(2.28-3.14)$ & $2.83(2.42-3.33)$ & $<0.001$ & $2.66(2.26-3.11)$ & $2.77(2.36-3.25)$ & $<0.001$ \\
\hline$A L T, U / L$ & $17.90(12.90-26.90)$ & $22.00(16.00-33.00)$ & $<0.001$ & $17.10(12.40-26.00)$ & $21.00(14.70-31.00)$ & $<0.001$ \\
\hline AST,U/L & $22.00(18.40-26.20)$ & 23.80 (20.00-28.00) & $<0.001$ & $21.60(18.10-26.00)$ & $23.00(19.00-28.00)$ & $<0.001$ \\
\hline BUN, mmol/L & $4.53(3.81-5.35)$ & $4.88(4.13-5.72)$ & $<0.001$ & $4.50(3.79-5.30)$ & $4.73(4.00-5.55)$ & $<0.001$ \\
\hline $\mathrm{Cr}, \mathrm{umol} / \mathrm{L}$ & $69.10(57.70-81.00)$ & $73.00(62.00-84.00)$ & $<0.001$ & $68.30(57.00-80.30)$ & $73.00(61.30-83.90)$ & $<0.001$ \\
\hline TyG index & $8.36(0.59)$ & $8.77(0.61)$ & $<0.001$ & $8.30(0.57)$ & $8.58(0.59)$ & $<0.001$ \\
\hline TyG-BMl & 194.49 (35.64) & 221.76 (36.36) & $<0.001$ & 191.08 (34.69) & $209.92(35.77)$ & $<0.001$ \\
\hline Family history of diabetes & $2358(2.21 \%)$ & $100(2.44 \%)$ & 0.330 & $1898(2.16 \%)$ & $310(2.51 \%)$ & 0.013 \\
\hline Smoking status & & & $<0.001$ & & & $<0.001$ \\
\hline Non & 5825 (5.46\%) & $291(7.09 \%)$ & & 4509 (5.13\%) & $840(6.80 \%)$ & \\
\hline Past & 1189 (1.11\%) & 47 (1.15\%) & & $935(1.06 \%)$ & $155(1.25 \%)$ & \\
\hline Current & 22,902 (21.46\%) & $665(16.21 \%)$ & & $18,877(21.46 \%)$ & $2322(18.80 \%)$ & \\
\hline Not recorded & $76,819(71.97 \%)$ & $3100(75.55 \%)$ & & 63,636 (72.35\%) & 9035 (73.15\%) & \\
\hline Drinking status & & & $<0.001$ & & & $<0.001$ \\
\hline Non & $736(0.69 \%)$ & 48 (1.17\%) & & 539 (0.61\%) & $100(0.81 \%)$ & \\
\hline Past & 5025 (4.71\%) & 189 (4.61\%) & & 3944 (4.48\%) & 604 (4.89\%) & \\
\hline Current & 24,155 (22.63\%) & 766 (18.67\%) & & 19,838 (22.55\%) & 2613 (21.15\%) & \\
\hline Not recorded & 76,819 (71.97\%) & 3100 (75.55\%) & & 63,636 (72.35\%) & 9035 (73.15\%) & \\
\hline
\end{tabular}

Abbreviations as in Table 1

TyG-BMI-related studies. In two recent TyG-BMIrelated studies, some scholars noted that TyG-BMI was related to a significantly higher risk of NAFLD and diabetes in these populations $[18,19]$. These findings suggest a special population dependence on the association of TyG-BMI with various metabolic diseases, among which more attention should be given to the evaluation of TyG-BMI in young and middle-aged people, women and non-obese people. Currently, the reasons for the specific population dependence of the association of TyG-BMI with multiple metabolic diseases are not clear, but the following points may require attention: (1) With the rapid development of society, urbanization and informatization, on-duty workers inevitably reduce their demand for physical activity, so they are more prone to metabolic problems [36]. (2) With the accelerated development of social informatization, young people have developed increasingly unhealthy living habits, which leads to a variety of metabolic problems prematurely [37, 38]. (3) Men and women have different body compositions; generally speaking, women have more fat mass, men have more lean mass [39], and BMI does not seem to 
Table 3 Cox regression analyses for the association between TyG-BMI and incident pre-diabetes in different models

\begin{tabular}{|c|c|c|c|c|}
\hline \multirow[t]{2}{*}{ TyG-BMI } & \multicolumn{4}{|l|}{$\mathrm{HR}, 95 \% \mathrm{Cl}$} \\
\hline & Unadjusted Model & Model I & Model II & Model III \\
\hline \multicolumn{5}{|l|}{ WHO } \\
\hline Multivariable Analysis (HR Per SD increase) & $1.89(1.85,1.94)$ & $1.42(1.38,1.47)$ & $1.39(1.32,1.47)$ & $1.40(1.32,1.47)$ \\
\hline \multicolumn{5}{|l|}{ TyG-BMI quartile } \\
\hline Q1 & Ref & Ref & Ref & Ref \\
\hline Q2 & $2.56(2.21,2.97)$ & $1.60(1.38,1.86)$ & $1.59(1.30,1.96)$ & $1.60(1.30,1.96)$ \\
\hline Q3 & $4.83(4.21,5.54)$ & $2.18(1.89,2.52)$ & $2.17(1.78,2.65)$ & $2.17(1.78,2.65)$ \\
\hline Q4 & $8.93(7.84,10.19)$ & $2.96(2.57,3.42)$ & $2.77(2.25,3.40)$ & $2.77(2.26,3.41)$ \\
\hline$P$-trend & $<0.0001$ & $<0.0001$ & $<0.0001$ & $<0.0001$ \\
\hline \multicolumn{5}{|l|}{ ADA } \\
\hline Multivariable Analysis (HR Per SD increase) & $1.54(1.52,1.56)$ & $1.27(1.24,1.29)$ & $1.23(1.18,1.27)$ & $1.23(1.18,1.27)$ \\
\hline \multicolumn{5}{|l|}{ TyG-BMI quartile } \\
\hline Q1 & Ref & Ref & Ref & Ref \\
\hline Q2 & $1.74(1.63,1.86)$ & $1.34(1.25,1.43)$ & $1.36(1.23,1.50)$ & $1.36(1.23,1.49)$ \\
\hline Q3 & $2.66(2.50,2.83)$ & $1.70(1.59,1.81)$ & $1.56(1.41,1.71)$ & $1.56(1.41,1.71)$ \\
\hline Q4 & $3.86(3.64,4.10)$ & $2.03(1.90,2.17)$ & $1.82(1.63,2.02)$ & $1.81(1.63,2.02)$ \\
\hline$P$-trend & $<0.0001$ & $<0.0001$ & $<0.0001$ & $<0.0001$ \\
\hline
\end{tabular}

Abbreviations: TyG-BMI triglyceride glucose-body mass index, HR hazard ratios, $C l$ confidence, ADA American Diabetes Association, WHO World Health Organization WHO: Model I adjusted for age, SBP, DBP, FPG, ALT and HDL-C; Model II adjusted for age, sex, SBP, DBP, FPG, TG, HDL-C, LDL-C, ALT, AST, BUN, Cr, drinking status and smoking status. Model III adjusted for age, sex, SBP, DBP, FPG, TG, HDL-C, LDL-C, ALT, AST, BUN, Cr, drinking status, smoking status, height and family history of diabetes

ADA: Model I adjusted for age, SBP, DBP, FPG, ALT and HDL-C; Model II adjusted for age, sex, SBP, DBP, FPG, TG, HDL-C, LDL-C, ALT, AST, BUN, Cr, smoking status, height and drinking status. Model II adjusted for age, sex, SBP, DBP, FPG, TG, HDL-C, LDL-C, ALT, AST, BUN, Cr, smoking status, height, drinking status and family history of diabetes

reflect the obesity information of the body very well [40]. In addition, Asian people are also prone to secondary metabolic problems, even if they are nonobese [41].

Notably, the mean age of the subjects in this study was relatively young. Some previous studies have shown that abnormalities in lipoprotein cholesterol, apolipoprotein and lipoprotein (a) are closely related to premature cardiovascular disease [42-44], among which IR is the main pathological mechanism leading to susceptibility to premature cardiovascular disease [45]. TyG-BMI is a compound index including glucose and lipids and has excellent IR recognition ability. Combined with the findings of this study and the age characteristics of the subjects, we believe that TyG-BMI may also be used to predict the risk of premature cardiovascular disease.
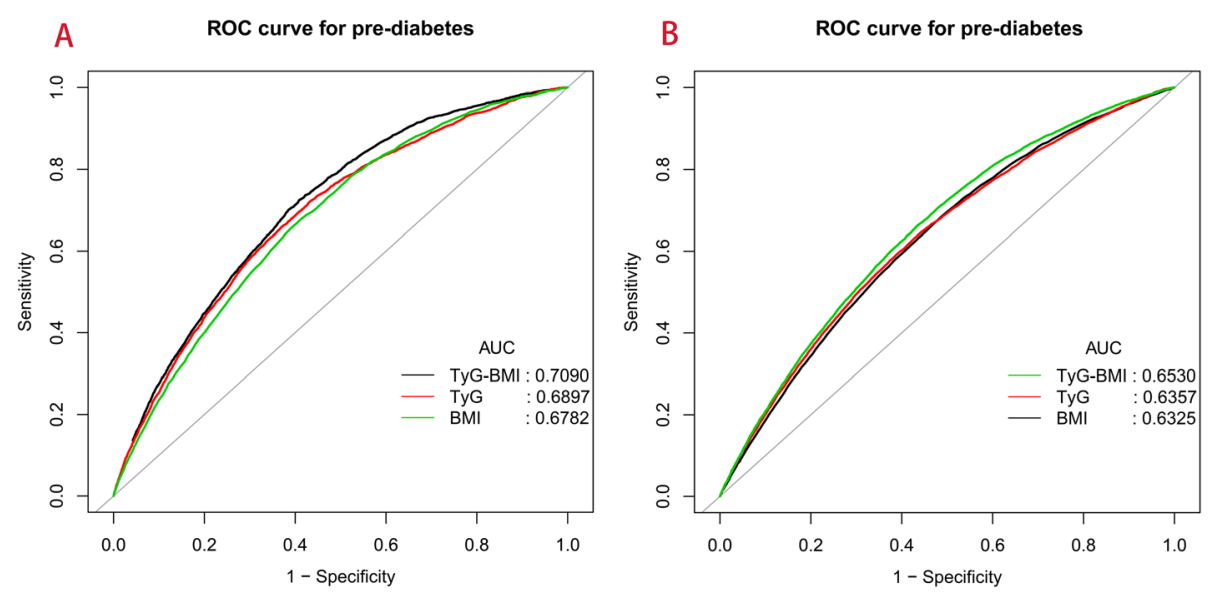

Fig. 3 Receiver operating characteristic curve analyses for predicting pre-diabetes (A: according to WHO diagnostic criteria; B: according to ADA diagnostic criteria). AUC: area under the curve; BMl: body mass index, TyG index: triglyceride-glucose index, TyG-BMl: triglyceride glucose-body mass index 
Table 4 Areas under the receiver operating characteristic curves for TyG-BMI, TyG index and BMI in identifying pre-diabetes

\begin{tabular}{|c|c|c|c|c|c|c|}
\hline & AUROC & 95\% Cl low & $95 \% \mathrm{Cl}$ upp & Best threshold & Specificity & Sensitivity \\
\hline \multicolumn{7}{|l|}{ WHO } \\
\hline TyG-BMI & 0.7090 & 0.7015 & 0.7165 & 200.0325 & 0.5913 & 0.7243 \\
\hline TyG index & 0.6897 & 0.6817 & 0.6977 & 8.5305 & 0.6314 & 0.6573 \\
\hline BMI & 0.6782 & 0.6703 & 0.6860 & 23.6950 & 0.5865 & 0.6785 \\
\hline \multicolumn{7}{|l|}{ ADA } \\
\hline TyG-BMI & 0.6530 & 0.6480 & 0.6580 & 193.4081 & 0.5657 & 0.6625 \\
\hline TyG index & 0.6357 & 0.6306 & 0.6409 & 8.3711 & 0.5727 & 0.6319 \\
\hline BMl & 0.6325 & 0.6274 & 0.6375 & 22.7950 & 0.5101 & 0.6870 \\
\hline
\end{tabular}

Abbreviations: AUROC Areas under the receiver operating characteristic curves, BMI body mass index, TyG index triglyceride glucose index, TyG-BMI triglyceride glucose-body mass index, ADA American Diabetes Association, WHO World Health Organization

\section{Study strengths and limitations}

This study has several strengths worth mentioning: (1) The sample size of this study is more than 100,000 and involves many regions in China, and the conclusion of the study is relatively objective and applicable to the Chinese population. (2) The study was analysed based on two different diagnostic criteria and reached a consistent conclusion, which can be considered relatively reliable. (3) The discovery of special population dependence on the risk of TyG-BMI-related metabolic diseases provides a new idea for health surveillance in the general population.
Some limitations need to be emphasized in this study: (1) The diagnostic criteria for pre-diabetes in this study were based on IFG; however, in the Asian population, the use of isolated IFG for pre-diabetes appears to result in a lower prevalence [46]. Considering that there is no unified diagnostic standard for pre-diabetes at present, this study also demonstrated the relationship between pre-diabetes and TyG-BMI at a lower prevalence rate, further implying that the conclusions of this study are relatively stable. (2) Due to the retrospective design of this study, in addition to some inherent statistical

Table 5 Stratified associations between TyG-BMI and pre-diabetes by age, sex, BMI and family history of diabetes

\begin{tabular}{|c|c|c|c|c|c|c|}
\hline Subgroup & $\begin{array}{l}\text { WHO } \\
\text { adjusted HR (95\% Cl) }\end{array}$ & $P$-value & $P$-interaction & $\begin{array}{l}\text { ADA } \\
\text { adjusted HR }(95 \% \mathrm{Cl})\end{array}$ & $P$-value & $P$-interaction \\
\hline Age (years) & & & $<0.0001$ & & & $<0.0001$ \\
\hline $18-29$ & $1.50(1.29,1.75)$ & $<0.0001$ & & $1.34(1.25,1.43)$ & $<0.0001$ & \\
\hline $30-39$ & $1.62(1.52,1.73)$ & $<0.0001$ & & $1.35(1.31,1.40)$ & $<0.0001$ & \\
\hline $40-49$ & $1.58(1.48,1.69)$ & $<0.0001$ & & $1.35(1.31,1.40)$ & $<0.0001$ & \\
\hline $50-59$ & $1.37(1.29,1.45)$ & $<0.0001$ & & $1.23(1.18,1.28)$ & $<0.0001$ & \\
\hline $60-69$ & $1.21(1.12,1.29)$ & $<0.0001$ & & $1.08(1.03,1.13)$ & 0.0031 & \\
\hline$\geq 70$ & $1.19(1.08,1.32)$ & $<0.0001$ & & $1.10(1.02,1.17)$ & 0.0086 & \\
\hline Sex & & & 0.0182 & & & $<0.0001$ \\
\hline Women & $1.49(1.41,1.57)$ & $<0.0001$ & & $1.40(1.35,1.44)$ & $<0.0001$ & \\
\hline Men & $1.38(1.33,1.44)$ & $<0.0001$ & & $1.18(1.15,1.20)$ & $<0.0001$ & \\
\hline BMI $\left(\mathrm{kg} / \mathrm{m}^{2}\right)$ & & & $<0.0001$ & & & $<0.0001$ \\
\hline$<18.5$ & $1.43(0.47,4.32)$ & 0.5252 & & $1.93(1.25,2.99)$ & 0.0030 & \\
\hline$\geq 18.5,<24$ & $1.84(1.66,2.04)$ & $<0.0001$ & & $1.51(1.44,1.59)$ & $<0.0001$ & \\
\hline$\geq 24,<28$ & $1.34(1.22,1.46)$ & $<0.0001$ & & $1.26(1.19,1.33)$ & $<0.0001$ & \\
\hline$\geq 28$ & $1.30(1.18,1.43)$ & $<0.0001$ & & $1.15(1.07,1.23)$ & 0.0001 & \\
\hline Family history of diabetes & & & 0.0625 & & & 0.4701 \\
\hline Yes & $1.20(0.99,1.44)$ & 0.0576 & & $1.31(1.19,1.45)$ & $<0.0001$ & \\
\hline No & $1.43(1.38,1.48)$ & $<0.0001$ & & $1.27(1.24,1.29)$ & $<0.0001$ & \\
\hline
\end{tabular}

Abbreviations: $B M I$ body mass index, TyG-BMI triglyceride glucose-body mass index, $H R$ hazard ratios, $C l$ confidence, ADA American Diabetes Association, WHO World Health Organization

The model adjusted for age, SBP, DBP, FPG, ALT and HDL-C;

Note: For age stratified analysis, the stratification variable was omitted from the model 
limitations, the historical data that our researchers were able to extract were also relatively limited. For example, this study lacked general measurement information such as waist circumference, neck circumference and hip circumference as well as information about chronic diseases and drug use of the subjects. Therefore, we were unable to evaluate the predictive performance of these simple measurement parameters for pre-diabetes in this study, and we were also unable to perform some subgroup analyses of comorbidities and drug therapy. (3) The follow-up period of this study was relatively short, and further follow-up studies are needed to further explore the causal association between the two. (4) According to the results of previous studies, IR may be an important factor in the association of TyG-BMI with pre-diabetes [15]. However, relevant IR information was not measured in this study; thus, the mechanism of the association between pre-diabetes and TyG-BMI needs to be verified by further studies. (5) As the present study was conducted in the Chinese population, whether TyGBMI can be used to assess the risk of pre-diabetes in other ethnic groups needs further study.

\section{Conclusion}

Overall, TyG-BMI is independently associated with prediabetes, whether using the ADA or WHO diagnostic criteria. TyG-BMI may be an accessible and supplementary monitoring method in the risk stratification management of patients with pre-diabetes. In addition, there is a special population dependence between TyG-BMI and many metabolism-related diseases. Therefore, we suggest that TyG-BMI screening should be performed routinely in non-obese people, women and people under 50 years old.

\section{Abbreviations}

TyG-BMI: Triglyceride glucose-body mass index; ADA: American Diabetes Association; WHO: World Health Organization; IFG: Impaired fasting glucose; FPG: Fasting plasma glucose; IR: Insulin resistance; HEC: Hyperinsulinemiceuglycemic clamp; AUROC: Area under the receiver operating characteristic curve; HR: Hazard ratios; Cl: Confidence intervals; KM: Kaplan-Meier; BMI: Body mass index; TC: Total cholesterol; TG: Triglyceride; LDL-C: Low density lipoprotein cholesterol; ALT: Alanine aminotransferase; AST: Aspartate aminotransferase; BUN: Blood urea nitrogen; Cr: Creatinine; HDL-C: High density lipoprotein cholesterol; TyG index: Triglyceride glucose index; MS: Metabolic syndrome; ROC: Receiver operating characteristic curve; NAFL D: Nonalcoholic fatty liver disease

\section{Supplementary Information}

The online version contains supplementary material available at https://doi. org/10.1186/s12944-021-01594-7.

Additional file 1.

\section{Authors' contributions}

Study design: YZ, CY-J. Data analysis and interpretation: YZ, RJ-Y, MB-K, MY, $M C-Z$ and $C Y-J$. Drafting the manuscript or revising it critically for important intellectual content: YZ, RJ-Y, MB-K, MY, MC-Z and CY-J. All authors read and approved the final manuscript.

\section{Funding}

Not applicable.

\section{Availability of data and materials}

The datasets that support the conclusions of this article can be found in the Dryad repository.

\section{Declarations}

Ethics approval and consent to participate

As the ethics committee of Rich Healthcare Group had authorized the previous study, the ethics committee of Jiangxi Provincial People's Hospital exempted the repeated application for ethical approval for this study (ethical review No. 2021-067)

\section{Consent for publication}

Not applicable.

\section{Competing interests}

No.

\section{Author details}

'Department of Cardiology, Jiangxi Provincial People's Hospital Affiliated to Nanchang University, Nanchang 330006, China. ${ }^{2}$ Department of Endocrinology, Jiangxi Provincial People's Hospital Affiliated to Nanchang University, Nanchang 330006, China. ${ }^{3}$ From the Jiangxi Cardiovascular Research Institute, Jiangxi Provincial People's Hospital Affiliated to Nanchang University, Nanchang 330006, China.

Received: 9 October 2021 Accepted: 4 November 2021

Published online: 14 November 2021

\section{References}

1. Tabák AG, Herder C, Rathmann W, Brunner EJ, Kivimäki M. Prediabetes: a high-risk state for diabetes development. Lancet. 2012;379(9833):2279-90.

2. Khetan AK, Rajagopalan S. Prediabetes. Can J Cardiol. 2018;34(5):615-23.

3. Alberti KG, Zimmet PZ. Definition, Diagnosis and classification of diabetes mellitus and its complications. Part 1: diagnosis and classification of diabetes mellitus provisional report of a WHO consultation. Diabet Med. 1998;15(7):539-53.

4. American Diabetes Association Classification and diagnosis of diabetes. Standards of medical care in diabetes-2018. Diabetes Care. 2018;41(Suppl 1):S13-27.

5. Yang W, Lu J, Weng J, Jia W, Ji L, Xiao J, et al. Prevalence of diabetes among men and women in China. N Engl J Med. 2010;362(12):1090-101.

6. $L i$ Y , Teng $D$, Shi $X$, Qin $G$, Qin $Y$, Quan $H$, et al. Prevalence of diabetes recorded in mainland China using 2018 diagnostic criteria from the American Diabetes Association: national cross sectional study. BMJ. 2020; 369:m997.

7. Plantinga LC, Crews DC, Coresh J, Miller ER 3rd, Saran R, Yee J, et al. Prevalence of chronic kidney disease in US adults with undiagnosed diabetes or prediabetes. Clin J Am Soc Nephrol. 2010;5(4):673-82.

8. Ziegler D, Rathmann W, Dickhaus T, Meisinger C, Mielck A, KORA Study Group. Prevalence of polyneuropathy in pre-diabetes and diabetes is associated with abdominal obesity and macroangiopathy: the MONICA/ KORA Augsburg surveys S2 and S3. Diabetes Care. 2008;31(3):464-9.

9. Gabir MM, Hanson RL, Dabelea D, Imperatore G, Roumain J, Bennett PH, et al. Plasma glucose and prediction of microvascular disease and mortality: evaluation of 1997 American Diabetes Association and 1999 World Health Organization criteria for diagnosis of diabetes. Diabetes Care. 2000;23(8): 1113-8.

10. Emerging Risk Factors Collaboration, Sarwar N, Gao P, Seshasai SR, Gobin R, Kaptoge S, et al. Diabetes mellitus, fasting blood glucose concentration, and risk of vascular disease: a collaborative meta-analysis of 102 prospective studies. Lancet. 2010;375(9733):2215-22. 
11. Abdul-Ghani MA, Tripathy D, DeFronzo RA. Contributions of beta-cell dysfunction and insulin resistance to the pathogenesis of impaired glucose tolerance and impaired fasting glucose. Diabetes Care. 2006;29(5):1130-9.

12. Færch K, Vistisen D, Pacini G, Torekov SS, Johansen NB, Witte DR, et al. Insulin resistance is accompanied by increased fasting glucagon and delayed glucagon suppression in individuals with Normal and impaired glucose regulation. Diabetes. 2016;65(11):3473-81.

13. DeFronzo RA, Tobin JD, Andres R. Glucose clamp technique: a method for quantifying insulin secretion and resistance. Am J Phys. 1979;237(3):E214-23.

14. Muniyappa R, Lee S, Chen H, Quon MJ. Current approaches for assessing insulin sensitivity and resistance in vivo: advantages, limitations, and appropriate usage. Am J Physiol Endocrinol Metab. 2008;294(1):E15-26.

15. Er LK, Wu S, Chou HH, Hsu LA, Teng MS, Sun YC, et al. Triglyceride glucosebody mass index is a simple and clinically useful surrogate marker for insulin resistance in nondiabetic individuals. PLoS One. 2016;11(3):e0149731.

16. Raimi TH, Dele-Ojo BF, Dada SA, Fadare JO, Ajayi DD, Ajayi EA, et al. Triglyceride-glucose index and related parameters predicted metabolic syndrome in Nigerians. Metab Syndr Relat Disord. 2021;19(2):76-82.

17. Li Y, You A, Tomlinson B, Yue L, Zhao K, Fan H, Zheng L. Insulin resistance surrogates predict hypertension plus hyperuricemia. J Diabetes Investig. 2021;12(11):2046-53

18. Wang R, Dai L, Zhong Y, Xie G. Usefulness of the triglyceride glucose-body mass index in evaluating nonalcoholic fatty liver disease: insights from a general population. Lipids Health Dis. 2021;20(1):77.

19. Wang X, Liu J, Cheng Z, Zhong Y, Chen X, Song W. Triglyceride glucosebody mass index and the risk of diabetes: a general population-based cohort study. Lipids Health Dis. 2021;20(1):99.

20. Chen Y, et al. Data from: association of body mass index and age with incident diabetes in Chinese adults: a population-based cohort study. Dryad Dataset. 2018. https://doi.org/10.5061/dryad.ft8750v.

21. Chen $Y$, Zhang XP, Yuan J, Cai B, Wang XL, Wu XL, et al. Association of body mass index and age with incident diabetes in Chinese adults: a populationbased cohort study. BMJ Open. 2018;8(9):e021768.

22. Wax Y. Collinearity diagnosis for a relative risk regression analysis: an application to assessment of diet-cancer relationship in epidemiological studies. Stat Med. 1992;11(10):1273-87.

23. Fitchett EJA, Seale AC, Vergnano S, Sharland M, Heath PT, Saha SK, et al. Strengthening the reporting of observational studies in epidemiology for newborn infection (STROBE-NI): an extension of the STROBE statement for neonatal infection research. Lancet Infect Dis. 2016;16(10):e202-13.

24. Vandenbroucke JP, von Elm E, Altman DG, Gøtzsche PC, Mulrow CD, Pocock $\mathrm{SJ}$, et al. Strengthening the reporting of observational studies in epidemiology (STROBE): explanation and elaboration. Int J Surg. 2014;12(12): $1500-24$.

25. Zhou BF, Cooperative Meta-Analysis Group of the Working Group on Obesity in China. Predictive values of body mass index and waist circumference for risk factors of certain related diseases in Chinese adults-study on optimal cut-off points of body mass index and waist circumference in Chinese adults. Biomed Environ Sci. 2002;15(1):83-96.

26. Hu FB. Globalization of diabetes: the role of diet, lifestyle, and genes. Diabetes Care. 2011:34(6):1249-57.

27. Danaei G, Finucane MM, Lu Y, Singh GM, Cowan MJ, Paciorek CJ, et al. National, regional, and global trends in fasting plasma glucose and diabetes prevalence since 1980: systematic analysis of health examination surveys and epidemiological studies with 370 country-years and 2.7 million participants. Lancet. 2011;378(9785):31-40.

28. Guerrero-Romero F, Simental-Mendía LE, González-Ortiz M, Martínez Abundis E, Ramos-Zavala MG, Hernández-González SO, et al. The product of triglycerides and glucose, a simple measure of insulin sensitivity. Comparison with the euglycemic-hyperinsulinemic clamp. J Clin Endocrinol Metab. 2010;95(7):3347-51.

29. Bastard JP, Lavoie ME, Messier V, Prud'homme D, Rabasa-Lhoret $R$. Evaluation of two new surrogate indices including parameters not using insulin to assess insulin sensitivity/resistance in non-diabetic postmenopausal women: a MONET group study. Diabetes Metab. 2012; 38(3):258-63.

30. Wen J, Wang A, Liu G, Wang M, Zuo Y, Li W, et al. Elevated triglycerideglucose (TyG) index predicts incidence of prediabetes: a prospective cohort study in China. Lipids Health Dis. 2020;19(1):226.

31. Ding J, Chen X, Bao K, Yang J, Liu N, Huang W, et al. Assessing different anthropometric indices and their optimal cutoffs for prediction of type 2 diabetes and impaired fasting glucose in Asians: the Jinchang cohort study. J Diabetes. 2020;12(5):372-84

32. Zheng S, Shi S, Ren X, Han T, Li Y, Chen Y, et al. Triglyceride glucose-waist circumference, a novel and effective predictor of diabetes in first-degree relatives of type 2 diabetes patients: cross-sectional and prospective cohort study. J Transl Med. 2016;14(1):260.

33. Ramdas Nayak VK, Nayak KR, Vidyasagar S, P R. Predictive performance of traditional and novel lipid combined anthropometric indices to identify prediabetes. Diabetes Metab Syndr. 2020;14(5):1265-72.

34. Valgimigli M, Gragnano F, Branca M, Franzone A, Baber U, Jang Y, et al. P2Y12 inhibitor monotherapy or dual antiplatelet therapy after coronary revascularisation: individual patient level meta-analysis of randomised controlled trials. BMJ. 2021;373:n1332.

35. Calabrò P, Gragnano F, Di Maio M, Patti G, Antonucci E, Cirillo P, et al. Epidemiology and Management of Patients with Acute Coronary Syndromes in contemporary real-world practice: evolving trends from the EYESHOT study to the START-ANTIPLATELET registry. Angiology. 2018;69(9): 795-802.

36. Pedersen BK, Saltin B. Exercise as medicine - evidence for prescribing exercise as therapy in 26 different chronic diseases. Scand J Med Sci Sports. 2015;25(Suppl 3):1-72.

37. American College of Obstetricians and Gynecologists' Committee on Adolescent Health Care. Committee opinion No. 653: concerns regarding social media and health issues in adolescents and young adults. Obstet Gynecol. 2016;127(2):e62-5

38. McMahon DM, Burch JB, Youngstedt SD, Wirth MD, Hardin JW, Hurley TG, et al. Relationships between chronotype, social jetlag, sleep, obesity and blood pressure in healthy young adults. Chronobiol Int. 2019;36(4):493-509.

39. Bredella MA. Sex differences in body composition. Adv Exp Med Biol. 2017; 1043:9-27.

40. Zou Y, Sheng G, Yu M, Xie G. The association between triglycerides and ectopic fat obesity: an inverted U-shaped curve. PLoS One. 2020;15(11): e0243068.

41. Kwon YM, Oh SW, Hwang SS, Lee C, Kwon H, Chung GE. Association of nonalcoholic fatty liver disease with components of metabolic syndrome according to body mass index in Korean adults. Am J Gastroenterol. 2012; 107(12):1852-8

42. Genest J Jr, McNamara JR, Ordovas JM, et al. Lipoprotein cholesterol, apolipoprotein A-I and B and lipoprotein (a) abnormalities in men with premature coronary artery disease. J Am Coll Cardiol. 1992;19(4):792-802.

43. Gragnano F, Fimiani F, Di Maio M, et al. Impact of lipoprotein(a) levels on recurrent cardiovascular events in patients with premature coronary artery disease. Intern Emerg Med. 2019;14(4):621-5.

44. Hopkins PN, Wu LL, Hunt SC, Brinton EA. Plasma triglycerides and type III hyperlipidemia are independently associated with premature familial coronary artery disease. J Am Coll Cardiol. 2005;45(7):1003-12.

45. Bansilal S, Farkouh ME, Fuster V. Role of insulin resistance and hyperglycemia in the development of atherosclerosis. Am J Cardiol. 2007; 99(4A):6B-14B.

46. Yip WCY, Sequeira IR, Plank LD, Poppitt SD. Prevalence of pre-diabetes across ethnicities: a review of impaired fasting glucose (IFG) and impaired glucose tolerance (IGT) for classification of Dysglycaemia. Nutrients. 2017; 9(11):1273.

\section{Publisher's Note}

Springer Nature remains neutral with regard to jurisdictional claims in published maps and institutional affiliations.

Ready to submit your research? Choose BMC and benefit from:

- fast, convenient online submission

- thorough peer review by experienced researchers in your field

- rapid publication on acceptance

- support for research data, including large and complex data types

- gold Open Access which fosters wider collaboration and increased citations

- maximum visibility for your research: over $100 \mathrm{M}$ website views per year

At $\mathrm{BMC}$, research is always in progress.

Learn more biomedcentral.com/submissions 